\title{
Resonant propagation of laser radiation in the upper ionosphere
}

\author{
Boris Shevtsov ${ }^{1}$, Vasily Bychkov, Andrey Perezhogin, and Ilya Seredkin \\ Institute of Cosmophysical Research and Radio Wave Propagation, FEB RAS
}

\begin{abstract}
A brief review report is presented on materials for studying the resonant propagation of laser radiation in the ionosphere excited by precipitations of charged particles. The results of research and perspectives for the development of scientific area are discussed. The solution for the problem of wave propagation in random fractal and resonant media, taking into account the backscattering features, which can be used for remote sensing of the characteristics of the excited ionosphere is considered.
\end{abstract}

The article is devoted to the analysis of lidar data obtained at wavelengths of 561.1 and 532 $\mathrm{nm}$ in the upper ionosphere above Kamchatka [1,2] in the fall of 2017, which was characterized by the appearance of light-scattering layers at heights of $200-450 \mathrm{~km}$ in magnetically quiet days.

To study the nature of such layers, it is first necessary to consider the reliability of their determination using a lidar, especially since in further observations such bright events as in the fall of 2017 were not repeated, possibly due to the onset of a minimum of solar activity. During the years of maximum solar activity, we observed this effect regularly, but only at a wavelength of $532 \mathrm{~nm}$.

In 2018, lidar observations were also carried out at wavelengths of 561.1 and $532 \mathrm{~nm}$. There were 33 successful nightly atmospheric sounding sessions, but there was no result in the fall of 2017. In 2019, observations were carried out only at a wavelength of $532 \mathrm{~nm}$, but with the readiness to turn on the sensing system at a wavelength of $561.1 \mathrm{~nm}$. It is possible that this waiting mode will have to be prolonged until the growth of solar activity resumes, in order to finally dispel doubts as to whether precipitation of thermal electrons is observed.

Since these precipitations occur on magnetically quiet days, it is obvious that they are a kind of memory effect of solar activity, a kind of relaxation process in the magnetosphere, capable of transforming short-wave solar radiation into thermal radiation due to resonant and nonlinear mechanisms, and this can already be used to explain the Maunder minimum and climate changes.

The light-scattering layers in the upper ionosphere can serve as indicators of precipitations of charged particles, which can affect both crews and equipment of spacecraft, as well as interfere with navigation and communication systems. Lidars provide a suffi ${ }^{1}$ ciently effective means to detect these precipitations.

1

Corresponding author: bshev@ikir.ru 
Solid-state lasers are used to measure and correct the parameters of the orbits of spacecraft, the resonance lines of the components of the ionosphere can fall into the study band. In this case, one can expect dispersion effects and their influence on the measurement results. 
At low atmospheric densities at high altitudes, the resonance scattering mechanism is decisive in the propagation of optical radiation, which, as shown by lidar observations, is effectively implemented in the upper ionosphere, and therefore it is necessary to study in details the features of this scattering. This article is devoted to this.

When analyzing lidar data in the upper ionosphere, two problems arose: an abnormally low signal at a wavelength of $561.1 \mathrm{~nm}$ against the expected one and increased oscillations of this signal at a decline after the maximum. It will be shown below that the cause of this signal behavior is the forced oscillations of atomic oxygen ions near a resonance whose width is much smaller than that of an atomic nitrogen ion.

Lidar parameters and working dipole transitions of the upper ionosphere components are given in tables 1 and 2 .

Table 1. Equipment

\begin{tabular}{|l|l|l|}
\hline \multicolumn{1}{|c|}{ Transmitter 1 } & \multicolumn{1}{|c|}{ Transmitter 2 } & \multicolumn{1}{c|}{ Receiver } \\
\hline Laser Nd:YAG Brilliant-B & Dye Laser TDL-90 & Telescope Diameter 60 cm \\
Pulse Energy 400 mJ & Pump Laser YG-982E & PMT Hamamatsu H8259-01 \\
Wavelength 532.08 nm & Pulse Energy 100 mJ & Photon Counters M8784-01, \\
Linewidth 0.04 nm & Wavelength 561.107 nm & Spatial resolution 1.5 km \\
Pulse duration 5 ns & Pulse duration 10 ns & Filters Bandwidth 1 nm \\
& Linewidth 0.025 nm & \\
\hline
\end{tabular}

Table 2. Dipole transitions of excited States of oxygen and nitrogen atomic ions [3] falling into the emission band of TDL-90 and Brilliant-b lasers.

\begin{tabular}{|l|l|l|l|l|l|l|l|l|l|}
\hline & $\begin{array}{c}\text { Compo } \\
\text { nent }\end{array}$ & $\begin{array}{c}\text { Wavelength } \\
\text { Air }(\mathrm{nm})\end{array}$ & $\begin{array}{c}\mathrm{A}_{\mathrm{ki}} \\
\left(\mathrm{s}^{-1}\right)\end{array}$ & Lower Level & Term J & Upper Level & Term & $\mathrm{J}$ \\
\hline 1 & O II & 561.1072 & $2.14 \mathrm{e}+06$ & $2 \mathrm{~s}^{2} 2 \mathrm{p}^{2}\left({ }^{1} \mathrm{~S}\right) 3 \mathrm{~s}$ & ${ }^{2} \mathrm{~S}$ & ${ }^{1 / 2}$ & $2 \mathrm{~s}^{2} 2 p^{2}\left({ }^{3} \mathrm{P}\right) 4 p$ & ${ }^{2} \mathrm{P}^{\circ}$ & $1 / 2$ \\
\hline 2 & NIII & 532.0870 & $5.68 \mathrm{e}+07$ & $2 \mathrm{~s} 2 \mathrm{p}\left({ }^{3} \mathrm{P}^{\circ}\right) 3 \mathrm{p}$ & ${ }^{2} \mathrm{D}$ & $5 / 2$ & $2 \mathrm{~s} 2 \mathrm{p}\left({ }^{3} \mathrm{P}^{\circ}\right) 3 \mathrm{~d}$ & ${ }^{2} \mathrm{~F}^{\circ}$ & $7 / 2$ \\
\hline 3 & NII & 532.0958 & $2.52 \mathrm{e}+07$ & $2 \mathrm{~s} 2 \mathrm{p}^{2}\left({ }^{4} \mathrm{P}\right) 3 \mathrm{p}$ & ${ }^{5} \mathrm{P}^{\circ}$ & 1 & $2 \mathrm{~s} 2 \mathrm{p}^{2}\left({ }^{4} \mathrm{P}\right) 3 \mathrm{~d}$ & ${ }^{5} \mathrm{P}$ & 2 \\
\hline
\end{tabular}

Here $\mathrm{OII}-$ ion $\mathrm{O}+$, NII - ion $\mathrm{N}+$, NIII - ion $\mathrm{N}++$. The lines presented in the table are selected taking into account their accordance to the laser radiation band. Doppler broadening of ion lines at ionospheric altitudes at a temperature of $800^{\circ} \mathrm{K}$ is about 0.004 $\mathrm{nm}$, which is less than the laser emission band, but much larger than the natural width of the ion lines, represented by $\mathrm{A}_{\mathrm{ki}}$ values.

The following results were obtained from lidar observations:

1. It was found that in all cases of electron precipitations into the ionosphere, anomalies were observed in the behavior of the horizontal and azimuthal (Eastern) components of the Earth's geomagnetic field.

2. The possibility of determining the energy spectrum of precipitated electrons by lidar method is shown. In all cases, the spectrum is defined as close to monoenergy with an energy of about $300 \mathrm{eV}$.

3. Lidar signals at wavelengths of 532 and $561 \mathrm{~nm}$ and their height profiles $\mathrm{N}(\mathrm{H})$ in the ionosphere $(100-500 \mathrm{~km})$ are analyzed on the basis of the resonance mechanism of interaction of excited atoms with laser radiation.

Consider anomalies in the geomagnetic field as the cause of precipitation of charged particles from the radiation belts and the formation of excited light-scattering layers in the ionosphere as a result. Figure 1 shows the variations of the azimuthal (Eastern) D and 
horizontal H components of the Earth's geomagnetic field, recorded on August 28, 2017 at the geomagnetic Observatory "Paratunka", and their Sq variations.

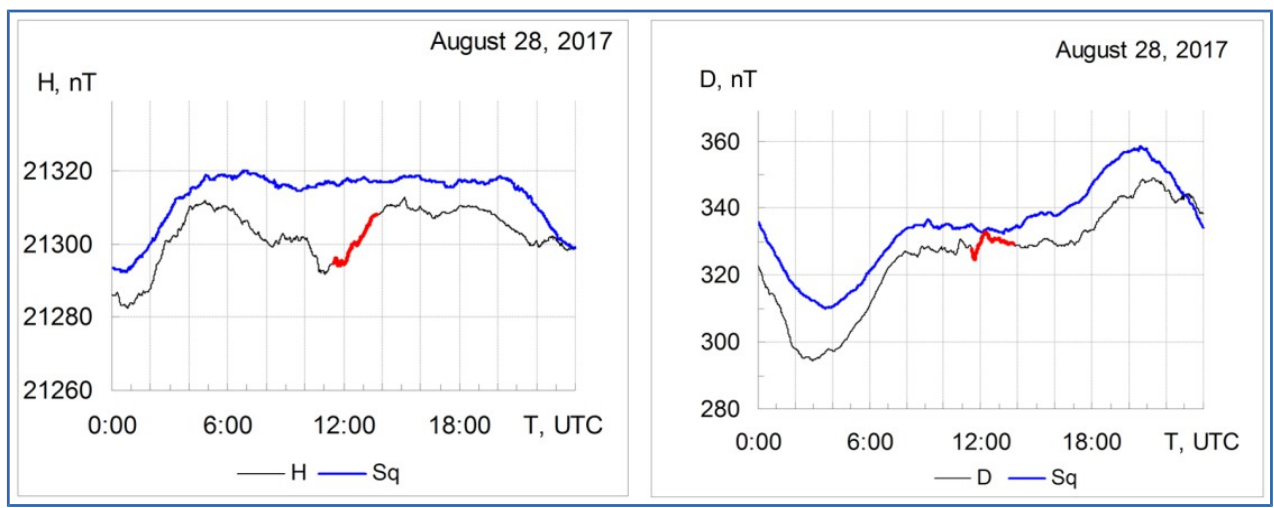

Fig. 1. The values of $\mathrm{D}$ and $\mathrm{H}$ component of the geomagnetic field and their 28.08.2017 Sq variation.

The values of Sq variations are obtained as the average value for the five quiet days of the previous month. The time of appearance of the resonance scattering signal during lidar observations is highlighted in red on the graphs.

Similar and also different kinds of anomalies was all days of the resonance scattering occurrence in the region at altitudes of $200-400 \mathrm{~km}$. It is clear that a change in any of component $\mathrm{D}$ or $\mathrm{H}$ will affect on the pitch-angle distribution of the particles in the force tube of the geomagnetic field, and may lead to violation of the capture conditions and precipitation of particles.

Let us consider the determination of the energy spectrum of electrons in precipitations. The upper part of figure 2 shows the distribution of the lidar signal received on September 5, 2017 [1]. The figure is based on lidar data with a 15-minute accumulation. The signal values are multiplied by $\mathrm{kH}^{2}$, where $\mathrm{H}$ is the height in $\mathrm{km}, \mathrm{k}=10^{-4}$, which corresponds to the geometric factor $\mathrm{H}^{2}$, and the value of the constant $\mathrm{k}$ is chosen so that at the height of 100 $\mathrm{km}$ the multiplied signal coincides with the actually registered one.

Analysis of lidar signals received in the fall of 2017 showed that they all have the following features:

- the signal at the wavelength of $532 \mathrm{~nm}$ is $20-30 \%$ larger than the signal at the wavelength of $561 \mathrm{~nm}$. The content of $\mathrm{O}+$ ions in the altitude region of $200-400 \mathrm{~km}$ is two orders of magnitude higher than the content of $\mathrm{N}+$ ions [4].

- the height of the maximum signal $(280-290 \mathrm{~km})$ does not correspond to the height of the maximum layer F2 of the ionosphere. After midnight it is usually (and on 5 September 2017 , according to ionosonde) in the region of $300-350 \mathrm{~km}$. The lifting of the layer at night due to a higher recombination rate in its lower part and maintaining the concentration in the upper part by the flow of ions from the plasmasphere, downward at night.

- on all total normalized signals at the wavelength of $561 \mathrm{~nm}$ there is a second local maximum in the region of $300-450 \mathrm{~km}$, which is less than $25 \%$ of the signal in the maximum. In this case, the values of signals at a wavelength of $532 \mathrm{~nm}$ monotonically decrease with increasing altitude from 280 to $450 \mathrm{~km}$. 


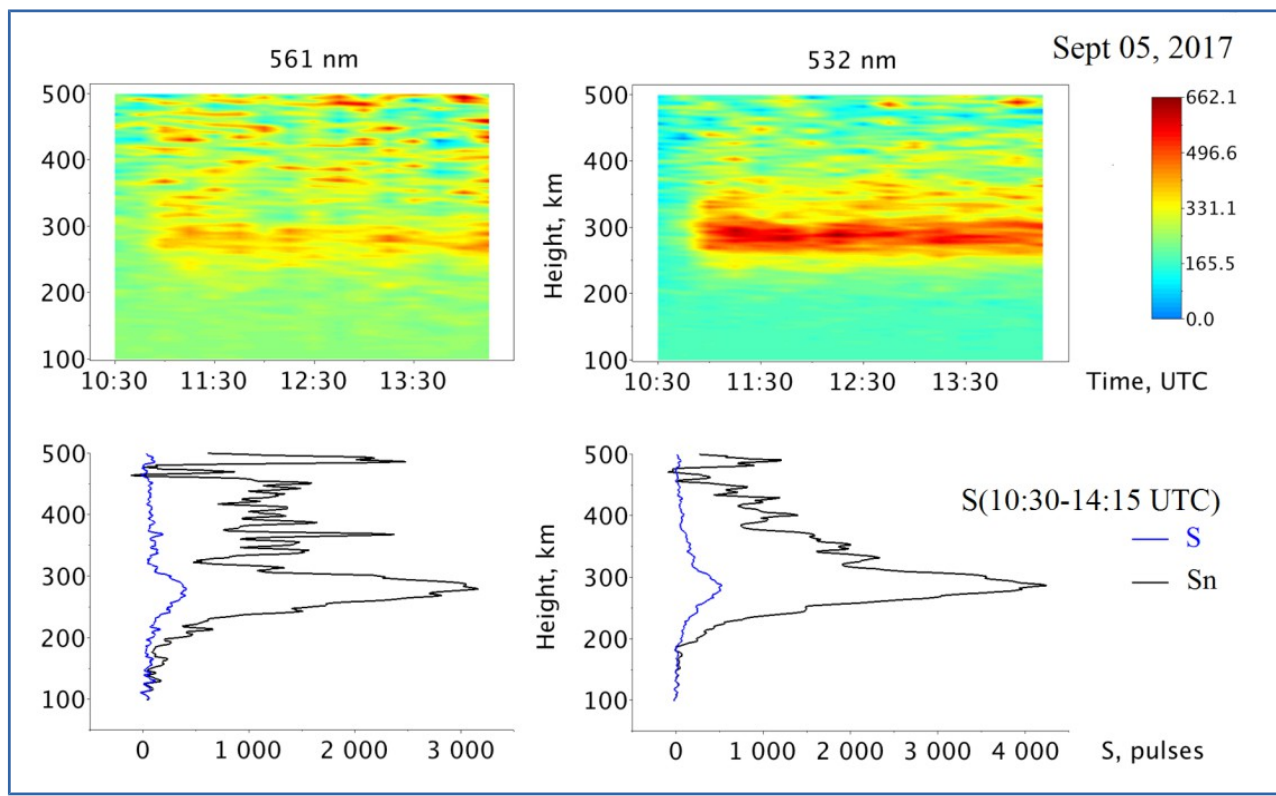

Fig. 2. Lidar "signal minus background" in the area of 100-500 km (top), the total signal S and normalized signal Sn (bottom), received on September 5, 2017.

The behavior of the total lidar signals at the night can be understood if we take into account the information given in figure 3. It presents a lidar signal at a wavelength of 532 $\mathrm{nm}$ (a) and the rate of ionization by a monoenergetic electron flux (b), calculated from the analytical approximation of the ionization rate given in $[5,6]$.

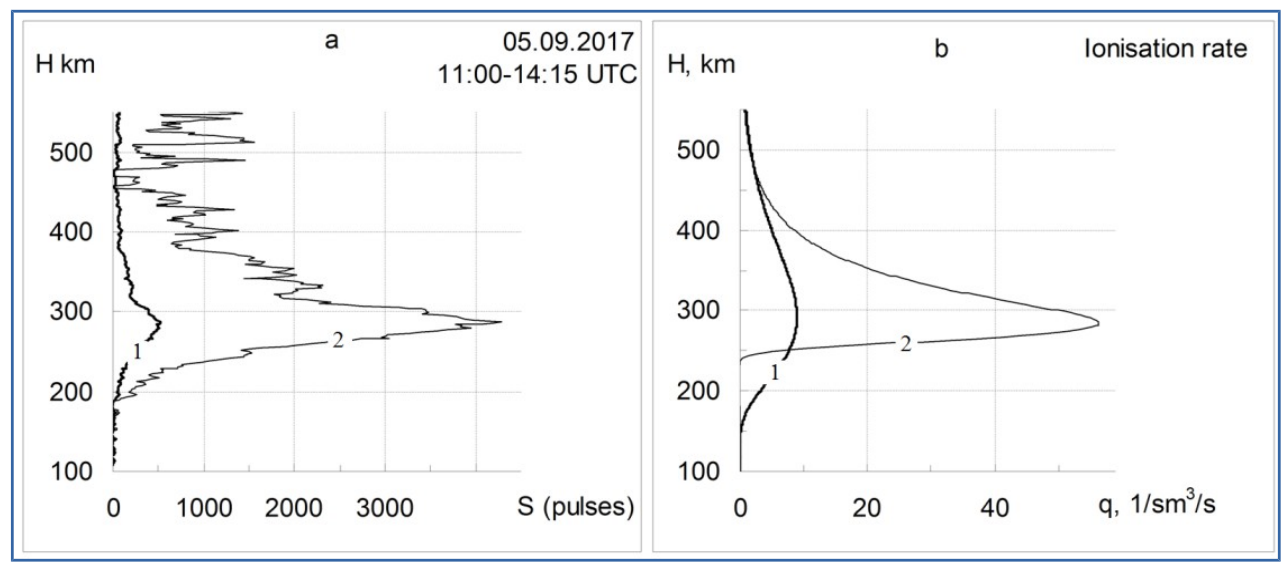

Fig. 3. Lidar signal at a wavelength of $532 \mathrm{~nm}$, received on September 5, 2017 (a), and electron flux with the Maxwell spectrum and the rate of ionization by a monoenergy electron beam (b).

In figure $3 \mathrm{a}$, curve 1 is the lidar signal $\mathrm{S}$, curve 2 is the signal $\mathrm{Sn}$ multiplied by the square of the height.

In figure $3 b$, curve 1 is the ionization rate for the electron flow with the Maxwell spectrum with a characteristic energy of $120 \mathrm{eV}$, curve 2 is for the monoenergy flow with an energy of $330 \mathrm{eV}$. 
The characteristic energy of the spectra of figure $3 \mathrm{~b}$ was chosen such that the height of the ionization maximum corresponded to the height of the maximum of the recorded lidar signal. The unknown value of the flux density was chosen to be $10^{8} \mathrm{~cm}^{-2} \mathrm{c}^{-1}$, which is typical for flows in the aurora zone.

From the comparison of figures $3 \mathrm{a}$ and $3 \mathrm{~b}$, it can be concluded that a monoenergetic electron flux with the energy close to $330 \mathrm{eV}$ was observed. The coincidence of the lidar signal shape and the ionization rate profile allows us to conclude that the main maximum of the lidar signal is formed as a result of scattering on atomic oxygen and nitrogen ions existing in the ionosphere and excited by electron precipitations.

Ionosonde data show that additional ionization is low, which suggests that the role of scatterers in lidar observations is mainly played by ions existing in the ionosphere and receiving additional excitation as a result of precipitations. Such processes are less energy intensive then ionization.

Ions of atomic oxygen and nitrogen in the F2 layer of the ionosphere arise due to numerous ionization reactions of $\mathrm{N} 2, \mathrm{O} 2, \mathrm{O}$. Atoms $\mathrm{N}$ are not included in this list, perhaps because they have a lower ionization capacity than $\mathrm{O}$ atoms.

In the lower atmosphere, where there are no ions, additional ionization becomes the main one.

On the decline of lidar signals (Fig. 2, Sn curve, 300-450 km in height), instability is manifested in their behavior, which is noticeably higher for the lidar signal of atomic oxygen ions than for the signal of atomic nitrogen ions. In intensity, these two signals differ little, while the concentration of atomic oxygen ions is two orders of magnitude higher than the concentration of atomic nitrogen ions. The approximate equality of intensities and the apparent instability of the signals in intensity requires explanation.

In this case, we are dealing with forced oscillations of two ions near the resonance at different quality factors, Fig. 2. For atomic oxygen ions, the quality factor of oscillations is greater than for atomic nitrogen ions (Table 1), therefore, the depth of the beats is greater.

The intensity of resonance scattering per unit volume is determined by the product of the scatterers concentration by the square of their width of the resonance line. For atomic oxygen and nitrogen ions, these products are the same, although the factors in them are different, which explains the approximate equality of the signal intensities at the maxima in Fig. 2.

Both problems that arose in the analysis of lidar data last year were resolved taking into account the resonant mechanism of light scattering by ions.

Reflections of electromagnetic signals by resonant media with fractal dispersion (a special form of the resonance line) were previously studied by modeling using the invariant immersion method [7]. The obtained solutions, which are characteristic of a beat resonance, can be used not only to justify the nature of the signals in Fig. 2, but also to restore the parameters of the scattering medium.

The proposed method of resonance scattering allows one to observe coherent oscillations of excited atomic oxygen and nitrogen ions in multi-kilometer volumes at an altitude of, for example, an ISS orbit of $400 \mathrm{~km}$ in order to study the dynamics of the ionosphere during precipitation of charged particles from radiation belts. But in general, we can talk about the possibility of developing optical technologies in the heights of the upper ionosphere.

The possibility of reliable detection by the method of two-frequency lidar sensing of unsteady light-scattering layers in the upper ionosphere is shown. Precipitations of 
electrons from radiation belts is not single reason for excitations. During magnetic storms in the aurora zone, this effect will be much brighter.

It is difficult to imagine the greater reliability of layer detection when resonance beats are observed in two sounding channels, and the intensity and frequency of signal beats are in certain proportions. On this, the problem of detecting excited light-scattering layer in the upper ionosphere can be considered solved.

\section{References}

1. V. V. Bychkov, A. S. Perezhogin, I. N. Seredkin, B. M. Shevtsov, Proc. SPIE, 10833-264 (2018)

2. V.V. Bychkov, I.N. Seredkin, E3S Web Conf., 62 (2018)

3. A. Kramida, Yu.Ralchenko, J. Reader, NIST ASD TEAM, NIST Atomic Spectra Database (ver. 5.5.2), Gaithersburg, MD (2018)

4. P. G. Richards, JGR, 116, A08307 (2011)

5. M. G. Deminov, Plasma Heliogeophysics (Moscow, Fizmatlit, 2008)

6. M. G. Deminov, V. V. Chegai, Geomagnetism and Aeronomy, 20 (1), 145-147 (1980)

7. A. S. Perezhogin, B. M. Shevtsov, 59, (2014) 